reproducible, meaning that only small numbers of patients would be required in trials that used FDG-PET to measure anti-inflammatory drug effect.

The authors performed FDG-PET and CT aortic and carotid imaging scans in 11 asymptomatic patients with atherosclerosis or elevated Framingham risk scores, and repeated the scans 2 weeks later. Mean arterial uptake of FDG in each aortic segment (ascending, arch, descending and abdominal) and either carotid artery was calculated and corrected for blood activity, providing arterial tissue-to-background ratios (TBRs). To test intraobserver and interobserver agreement, an experienced reader analyzed all first scans twice at least 1 month apart and these scans were also independently analyzed by a second reader.

There was no significant difference in TBR values between the first and second scan in any aortic segment or in either carotid artery, indicating that there was little spontaneous shortterm variation in FDG signal. The correlation coefficients for interobserver and intraobserver agreement, and for intrascan variation, were $\geq 0.8$ in all arterial regions except the aortic arch. The aortic and carotid TBR values had small standard deviations, suggesting that small numbers of patients would be required in clinical trials in order to show changes in TBR outside the range of normal variability.

Original article Rudd JHF et al. (2007) 18Fluorodeoxyglucose positron emission tomography imaging of atherosclerotic plaque inflammation is highly reproducible: implications for atherosclerosis therapy trials. J Am Coll Cardiol 50: 892-896

\section{Benefits of fondaparinux over enoxaparin in patients with renal dysfunction: OASIS 5 data}

Recent data have shown that fondaparinux, a selective factor $\mathrm{Xa}$ inhibitor, is noninferior to enoxaparin for short-term prevention of adverse cardiovascular outcomes in patients with non-ST-segment elevation acute coronary syndromes (ACS). Fondaparinux is also associated with a reduced incidence of major bleeding compared with enoxaparin; major bleeding is independently associated with increased mortality in this population. As renal dysfunction also increases the risk for major bleeding events, Fox et al. have tested the hypothesis that the benefits of fondaparinux over enoxaparin are greatest in those patients with the most-severe renal dysfunction.

The analysis included 19,979 patients from the OASIS 5 trial who presented with nonST-segment elevation ACS and had serum creatinine measured at baseline. In this trial, patients were randomized to received either fondaparinux $(2.5 \mathrm{mg} /$ day) or enoxaparin ( $1 \mathrm{mg} / \mathrm{kg} /$ day) for2-8 days. At 9 days, fondaparinux was noninferior to enoxaparin in terms of a composite end point of death, myocardial infarction or refractory ischemia. No significant differences in efficacy were observed between the two agents when patients were grouped by quartiles of estimated glomerular filtration rate (GFR).

Overall, the 9-day risk of major bleeding events was lower with fondaparinux than with enoxaparin (2.1\% vs $4.1 \%)$. This reduction in risk was observed in all quartiles of GFR, and was most pronounced in those individuals in the lowest quartile (GFR $<58 \mathrm{ml} / \mathrm{min} / 1.73 \mathrm{~m}^{2}$ ). These differences persisted at 30 and 180 days. Fondaparinux might, therefore, offer considerable benefits over enoxaparin in patients presenting with non-ST-segment elevation ACS who have poorly preserved renal function.

Original article Fox KAA et al. (2007) Influence of renal function on the efficacy and safety of fondaparinux relative to enoxaparin in non-ST-segment elevation acute coronary syndromes. Ann Intern Med 147: 304-310

\section{Dronedarone successfully maintains sinus rhythm in patients with atrial fibrillation}

Amiodarone is an effective antiarrhythmic agent, but it can have potentially serious adverse effects. Two identical multicenter trials were conducted to assess the efficacy of dronedarone, a potential alternative to amiodarone, for maintaining sinus rhythm in patients with atrial fibrillation (AF) or flutter. In total, 409 patients were randomized to placebo and 828 to dronedarone ( $400 \mathrm{mg}$ orally twice daily). The mean age of participants was 63 years.

After 12 months, fewer patients taking dronedarone had experienced a recurrence of $\mathrm{AF}$ than had those taking placebo $(64.1 \%$ vs $75.2 \%$; hazard ratio $0.75,95 \% \mathrm{Cl} 0.65-$ $0.87 ; P<0.001)$. The time to recurrence was more than twice as long among those taking the drug than among those taking placebo 